\title{
Diffusion-Based MR Methods for Bone Structure and Evolution
}

\author{
E.E. Sigmund,${ }^{1}$ H. Cho,${ }^{1}$ P. Chen,${ }^{1}$ S. Byrnes,${ }^{1}$ Y.-Q. Song, ${ }^{*}{ }^{\star}$ X.E. Guo, ${ }^{2}$ and T. R. Brown ${ }^{2}$
}

\begin{abstract}
A new approach to MR trabecular bone characterization is presented. This method probes the diffusion of spins through internal magnetic field gradients due to the susceptibility contrast between the bone and water (or marrow) phases. The resulting spin magnetization decay encodes properties of the underlying structure. This method, termed decay due to diffusion in the internal field (DDIF), is well established as a probe of pore size and structure. In the present work its application is shown for in vitro experiments on excised bovine tibiae samples. A comparison with pulsed field gradient (PFG) measurement of restricted diffusion shows a strong correlation of DDIF with the surface-to-volume ratio (SVR) of bones. Calculation of the internal magnetic field within the bone structure also supports this interpretation. These NMR measurements compare well with the image analysis from microscopic computed tomography $(\mu \mathrm{CT})$. The SVR is not accessible in the clinically standard densitometry measurements, and provides vital information on bone strength and therefore on its fracture risk. The DDIF and PFG methods derive this information from a straightforward pulse sequence that does not employ either high applied field gradients or microimaging, and thus may have clinical potential. Magn Reson Med 59:28-39, 2008. (c) 2007 WileyLiss, Inc.
\end{abstract}

Key words: trabecular bone; diffusion; internal field gradient; surface-to-volume ratio; DDIF

Osteoporosis is a disorder of the skeleton in which bone strength is abnormally weak and susceptible to fractures from minor trauma. Therapeutic treatment of osteoporosis is under intense development. Current diagnostics of osteoporosis using dual X-ray measurement of bone density do not entirely predict fracture risk, because the internal bone structure, apart from the bone density, contributes significantly to the mechanical strength and thus fracture risk (1,2). Such bone structure is routinely characterized by microscopic computed tomography ( $\mu \mathrm{CT}$ ) with resolution down to $10 \mu \mathrm{m}$ for small samples. However, it is not available for in vivo examination due to the high radiation dose. Recent efforts to achieve high-resolution 3D images of the trabecular architecture using magnetic resonance imaging (MRI) are very promising. However, it is difficult to drastically improve its resolution far beyond the current

1Schlumberger-Doll Research, Cambridge, Massachusetts, USA

${ }^{2}$ Department of Biomedical Engineering, Columbia University, New York, New York, USA.

E.E. Sigmund is now at the Department of Radiology, New York University, New York NY, USA.

Grant sponsor: National Institutes of Biomedical Imaging and Bioengineering; Grant number: EB003869-01.

*Correspondence to: Yi-Qiao Song, Schlumberger-Doll Research, 1 Hampshire Street, Cambridge, MA 02139. E-mail: ysong@slb.com

Received 19 September 2006; revised 26 February 2007; accepted 3 April 2007

DOI 10.1002/mrm.21281

Published online in Wiley InterScience (www.interscience.wiley.com).

(c) 2007 Wiley-Liss, Inc. levels $(\sim 100 \mu \mathrm{m})$ in clinical implementation, primarily due to the clinically allowed MRI scan time.

This article describes a different approach to the characterization of bone architecture compared to the highresolution imaging approach. An NMR technique is used that is well-established in inorganic porous media to obtain statistical properties of the trabecular structure. This technique, referred to as decay from diffusion in an internal field (DDIF), can obtain pore-structure characteristics (such as the pore-size distribution (3)) at a resolution of about $1 \mu \mathrm{m}$. In vitro DDIF data on bovine trabecular bone samples show a clear correlation with bone strength. This trend correlates well with measurements of the surface-tovolume ratio (SVR) using a pulsed field gradient (PFG), demonstrating the DDIF is also sensitive to the SVR of bones. This interpretation is further understood via theoretical calculations of the internal magnetic field. Both DDIF and PFG data exhibit two regimes at high and low bone strength with a contrasting correlation. These two regimes are interpreted as two types of bone-weakening behaviors: one dominated by the loss of bone density at the low yielding strength, and the other by the change of bone architecture at the high yielding strength. DDIF and PFG can be implemented as a simple modification of several clinical MR modalities for potential clinical application in osteoporosis diagnosis and the development of bonegrowth drugs.

\section{BACKGROUND}

\section{Current Methods}

Many different MR techniques are applied to the characterization of trabecular bone (4). Perhaps the most common MR methods for bone measurement fall into two main categories: susceptibility contrast and microscopic imaging. In the former case, the broadening $\left(1 / T_{2}^{\prime}\right)$ of the resonance line (or equivalently, the shortening of the free induction decay (FID)) due to the magnetic susceptibility contrast between the bone matrix and the intervening marrow is measured (5-7). This broadening depends on the bone architecture, which in turn determines the bone strength $(5,8)$. The second category consists of performing high-resolution microscopic MRI. Ex vivo and in vitro research studies of this type can achieve a resolution that is competitive with $\mu \mathrm{CT}(9-13)$. In vivo imaging is presently at a resolution near the length scale of the trabecular $\mathrm{rod} /$ plate separation $(14,15)$ and is therefore combined with subvoxel processing to refine the structure (16). From this more direct measurement of bone structure, topological parameters are derived that have been found to relate to bone strength (12).

Other MR techniques are being actively pursued as well. One example is ${ }^{31} \mathrm{P}$ imaging, which probes nuclei con- 
tained in the solid bone matrix (17). Another method employs the dipolar demagnetizing field to probe pore sizes in trabecular bone samples (18). A related method employing the distant dipolar field and its modulation due to internal magnetic field gradients has been applied to the trabecular bone system (19). All of these methods have seen significant development in the research community but have yet to become the clinical standard, for various reasons. For example, $T_{2}^{\prime}$ may have contributions from field variation over long length scales that are not directly related to trabecular structure. High-resolution imaging requires a long scan time and sophisticated subvoxel processing to obtain results, and is problematic for spine or hip imaging. The DDIF method applied to bone structure plays an intermediate role between the extremes of linewidth and full imaging approaches. To assist in the interpretation of the DDIF data, PFG diffusometry experiments can be performed as a function of both diffusion time and diffusion-sensitizing gradient orientation. This information allows an unambiguous determination of bone SVRs as a function of orientation, and thus probes the bone structure's scale and anisotropy.

In this work a brief review of trabecular bone structure and its evolution will be given first. Next, the DDIF method and how it relates to the more common PFG measurement will be described in detail. Several structural parameters derived from high-resolution $\mu \mathrm{CT}$ images of our sample set (e.g., the mean intercept length [MIL]) will also be described. Internal field calculations based upon this microscopic structure have also been performed and will be used to further understand the NMR data. The Results section will show data from each of these techniques and their correlations. Finally, the comparison of these techniques and the potential for clinical application will be discussed.

\section{Trabecular Bone Structure}

The mechanical strength of a bone specimen, and thus its risk of fracture, depend on several factors. There are two types of bone: cortical bone (a dense, microporous outer shell) and trabecular or cancellous bone (a more porous, spongy combination of rods and plates found near joints). Most of the load-bearing capability is attributed to the trabecular bone region, and the remodeling of trabecular bone structure is significantly affected by the mechanical forces it experiences, according to a relationship known as Wolff's law (20).

One very important factor in bone strength is the amount of bone material, which is represented by various parameters, such as the bone volume fraction (BVF) and porosity, and the most common clinical parameter, bone mineral density (BMD). However, it has been shown that densitometry alone cannot account for nearly half of the fracture risk $(1,2)$ and that the details of the trabecular bone structure could be important.

Trabecular bone consists of combinations of rods and plates with thicknesses/diameters on the scale of $100 \mu \mathrm{m}$ in an interconnected network. Bone structure changes are governed at the cellular level by osteoclasts, which control bone erosion, and osteoblasts, which control bone formation. If the overall remodeling performed by these two factions is dramatically imbalanced, as is postulated to occur in osteoporosis, serious weakening can occur in the trabecular bone network. These changes have been extensively characterized in vivo and ex vivo within and between different human subjects as a function of both age and mechanical strength using $\mu \mathrm{CT}(12,21)$ or MRI $(5,14,15)$. Structural indices, such as the mean trabecular thickness, mean trabecular separation, and trabecular number, can be calculated from microscopic images, and a wide variety of indices beyond these have been proposed to highlight structure, anisotropy, or correlation with strength (13). Many studies have shown that the parameter with the largest correlation with weakening is the trabecular number (also known as the trabecular plate density or surface density), or the number of trabeculae per unit length in a given direction. Trabecular widths are also known to decrease with age, but typically less dramatically than the trabecular number and not sufficiently to explain the weakening in most cases $(15,21)$. This discrepancy is one of the motivations for developing a widely accepted model of bone degradation (22). This model rationalizes the fact that the only explanation for the observed progression in bone structure is not a uniform thinning of trabecular plates, but a removal of entire plates with a consequent loss in connectivity. This removal is postulated to begin through a period of overactive osteoclastic resorption (bone removal) on a trabecular plate that consumes the entire thickness at a certain point, opening a hole or perforation that connects previously isolated regions of marrow. These perforations grow until eventually all that remains of the original plate is rods that connect to other adjacent plates. The trabecular number parameter that tracks this process is closely related to the SVR of the structure (23). The present work will show two NMR techniques that can probe this quantity without high-resolution imaging.

Another important feature of trabecular bone is its anisotropy $(5,11,19)$. Since many bones are under anisotropic mechanical load, their structure and development show anisotropy along the load direction. This anisotropy is often quantified with a fabric tensor that describes both the amount of anisotropy and its orientation through microscopic image measures, such as the MIL $(9,11)$, density autocorrelation lengths (24), and digital topology-based orientation analysis (DTA-O) (25). Models have been developed that relate these tensor elements to mechanical elastic moduli (26). Changes in anisotropy have also been observed to depend on the subject's stage of development (11). Both of the NMR techniques offered here are ideally suited to measure this anisotropy. In the PFG case, the diffusion-sensitizing gradient can be arbitrarily oriented. Also, the internal gradients are preferentially generated along the applied field direction, as has been noted in many NMR linewidth studies both in vitro and in vivo $(5,7,10)$. Thus, DDIF can also be sensitive to anisotropy.

\section{DDIF Concept}

Consider a bone consisting of a solid matrix and a waterfilled pore space. When this sample is place in a uniform magnetic field, a nonuniform field, $B^{i}$ (called the internal field), naturally appears inside the pore space due to the 
difference in the magnetic susceptibilities of the two phases. Because it is produced by the bone structure, this internal field carries a fingerprint of that structure. One of the key features of this field is that its spatial variation occurs primarily over the pore length for low-porosity materials (27). The first step of DDIF is to set up a spatial magnetization profile $(\mathrm{m})$ mimicking that of $B^{i}$ (for example, positive magnetization in regions where $B^{i}$ is higher, and negative magnetization where $B^{i}$ is lower than the average field). After this initial setup, the molecules diffuse in the pore space. The movement of spins from positive- $B^{i}$ regions to negative- $B^{i}$ regions causes a reduction of the magnetization profile. The DDIF experiment measures the time scale of this process and thus determines pore geometry. For the high-porosity $(80 \%)$ case of trabecular bone, the gradients mostly exist near the trabecular surfaces. Thus, the signal from near the trabecular surfaces exhibits enhanced decay compared to signal from the center of pores. As a result, DDIF in bones is sensitive to the SVR.

The DDIF experiment consists of a stimulated-echo pulse sequence and a reference scan to measure and separate the effect of spin-lattice relaxation. The pulse diagrams for these two steps are shown in Fig. 1. Details of the experiments were previously discussed in Ref. 28. The first and third periods of this sequence $\left(t_{e}\right)$ are the spatial encoding and decoding periods, and the middle period $\left(t_{D}\right)$ is the diffusion period. The encoding time $t_{e}$ is chosen so that the phase modulation $\Phi=\gamma B^{i} t_{e}$ across an average pore from the internal field $B^{i}$ is significant, e.g., $\Phi \sim \pi$. For fixed $t_{e}$, a series of echo amplitudes for variable diffusion times $t_{D}$ are measured. The decay of these amplitudes typically shows multiexponential behavior from the distribution of gradient magnitudes in the sample and is analyzed via Laplace inversion. The resulting magnitudes $A_{n}$ for different decay times $\tau_{n}$ form a spectrum that is herein referred to as a DDIF spectrum. Though the properties of this spectrum will by design depend on the sample structure, there are useful common behaviors that have been recognized in the development of the DDIF technique. One of these is a "weak encoding regime" in which the DDIF spectral intensity dominated by internal gradients (the "diffusion modes") scales linearly with $t_{e}$. This
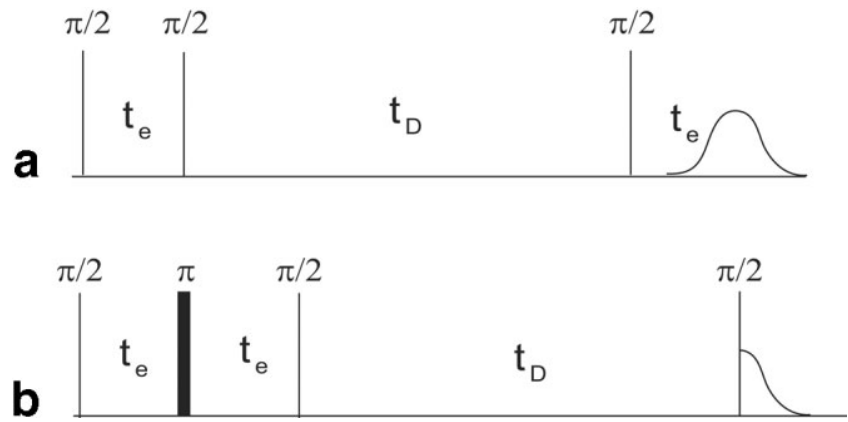

FIG. 1. DDIF (a) and reference (b) scan pulse sequences. The tipping angles of the pulses are marked as $\pi$ and $\pi / 2 . t_{e}$ and $t_{D}$ are the encoding and diffusion time periods, respectively. An echo signal is detected for DDIF, and an FID is detected for the reference sequence. behavior can be motivated by a mode expansion solution to the Bloch-Torrey equation for the magnetization evolution $(28,29)$. More intuitively, weak encoding corresponds to the range of $t_{e}$ values for which the phase modulation is small $(\Phi<1)$. In low-porosity samples with significantly extended gradients, this regime allows an estimation of the pore size distribution. The present work is limited to the weak encoding regime. While many theoretical and experimental treatments of diffusion through susceptibility-induced gradients exist in the study of porous media and biological tissue (30-35), the uniqueness of the DDIF approach is in the quantitative control and analysis of this structural encoding mechanism.

\section{MATERIALS AND METHODS}

\section{Sample Preparation}

Freshly frozen bovine proximal tibiae were obtained from a local slaughterhouse (Green Village Packing Co., Green Village, NJ) and cut into 8-mm-diameter and 40-mm-long cylinders along the principal orientation of the trabeculae, and then cleaned of their marrow. The ends of the specimens were glued in threaded brass end caps for mechanical testing. After the tests were completed, the middle 8-mm section was cut out for use as an NMR sample. The cylinder orientation was approximately the load-bearing axis of the parent tibiae. A detailed description of the procedure is provided elsewhere (36). Prior to the NMR measurements, the cleaned marrow space was saturated with water.

\section{Mechanical Testing}

Uniaxial compression tests were performed using a servohydraulic material testing machine (MTS810; MTS, Minneapolis, MN, USA). The stress-strain curve was measured for each sample to determine the yield stress and Young's modulus. A detailed description of the procedure is provided elsewhere (36).

\section{NMR DDIF Experiments}

All NMR experiments were performed in a $2 \mathrm{~T}\left({ }^{1} \mathrm{H} 85 \mathrm{MHz}\right)$ magnet (Nalorac Cryogenics) with a Biospec console (Bruker Biospin). The orientation of the applied field was along the cylindrical axis of each sample. In order to eliminate field variations due to the finite size of the sample, susceptibility-matched plugs (Wilmad Glass) were placed above and below the bone sample. DDIF and reference signals were collected for a range of logarithmically spaced diffusion times $t_{D}$ from $1 \mathrm{~ms}$ to $10 \mathrm{~s}$, and a range of encoding times $t_{e}$ from 5 to $30 \mathrm{~ms}$. Each dataset was processed as follows to produce a Laplace inversion spectrum: First, the reference data $R$ was subtracted from the DDIF data $E$ with a proportionality constant $a_{0}$ to remove the relaxation contribution (28):

$$
E_{s}=E-a_{0} R
$$

The maximum value of $a_{0}$ satisfying the criterion that the inversion of $E_{s}$ should contain only positive amplitudes was used in Eq. [1]. The Laplace inversion procedure was 
applied to the subtracted amplitudes $E_{s}$, giving a spectrum of decay times $A(T)$. The dependence of the DDIF spectra on the encoding time for these samples was qualitatively consistent with previous work (29) showing a weak encoding regime in which the integrated spectral weight scales with $t_{e}$. For sample comparisons, a representative encoding time for each sample was chosen empirically at the maximum point observed within the weak encoding regime, which was $t_{e}=30 \mathrm{~ms}$ except for three samples (which used $25 \mathrm{~ms}, 25 \mathrm{~ms}$, and $17 \mathrm{~ms}$ ). In all cases the signal had evidently saturated as a function of $t_{e}$.

The uncertainty of the DDIF spectrum was estimated as follows: First, the standard deviation (SD) $\sigma$ of the difference between the raw magnetization data $E_{s}$ and its best fit was measured. The Laplace inversion was repeated 20 times with newly generated Gaussian noise of identical amplitude $\sigma$ added to the original data $E_{s}$. The SD of each point in the spectrum obtained from these repetitions is shown as an error bar on all Laplace inversion spectra in this work. For the sake of consistency, all spectra shown in this work were obtained with the same regularization parameter (37), which was chosen as the average of the independently optimized values. These individual values were determined by a "heel" approach, wherein the maximum value of $\alpha$ preceding a dramatic increase in the fit $\chi^{2}$ is used.

\section{NMR PFG Experiments and Restricted Diffusion}

In the PFG experiments the $z$-axis gradient was oriented along the applied field and the sample's cylindrical axis. A stimulated-echo sequence with internal field compensation (38) was used to measure the apparent diffusion coefficient (ADC) along each gradient direction and for each diffusion time (see Fig. 2). For a given diffusion time, a single ADC was determined by measuring the echo decay for fixed sequence timing and variable diffusion gradient strength $G$. The diffusion-weighting factor for this sequence, calculated from the gradient waveform shown in Fig. 2 (38,39), was

$$
b=\gamma^{2} G^{2}(2 \delta)^{2}\left(\Delta+\frac{3}{2} \tau-\frac{1}{6} \delta\right) .
$$

The decays were all found to be single exponential in form over at least an order of magnitude. For every sample, ADCs were measured along both the transverse $(y)$ and longitudinal $(z)$ directions for a range of diffusion times

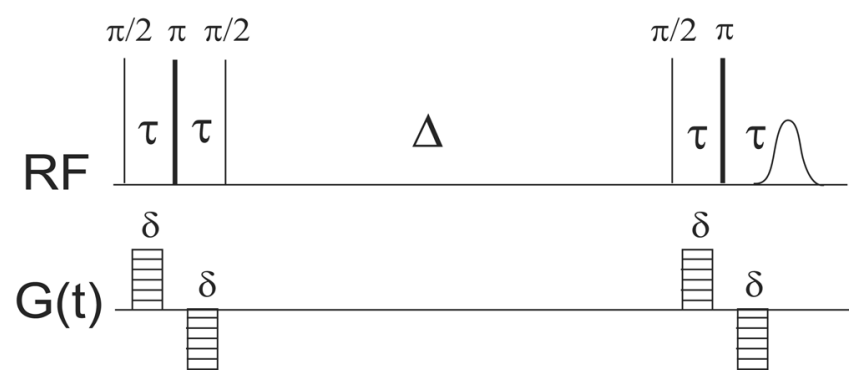

FIG. 2. RF and gradient pulse sequences for the PFG time-dependent diffusion measurement. from $\Delta=200 \mathrm{~ms}$ to $3 \mathrm{~s}$, with $\tau=2.5 \mathrm{~ms}$ and $\delta=2 \mathrm{~ms}$. A similar set of diffusion coefficients were measured in a bulk water sample under the same conditions. The timedependent diffusion coefficients $D(t)$ for bone were normalized to the average of bulk water values measured at the same time points.

The behavior of $D(t)$ in a restricted diffusion medium is well understood (40). The essence of this method is that diffusion is only restricted near the surface and the fractional volume affected is approximately $S / V \times \sqrt{D_{0} t}$, where the diffusion length $\sqrt{D_{0} t}$ is approximately the thickness of the affected region. As a result, the measured ADC will decrease as diffusion time $t$ increases when $\sqrt{D_{0} t}$ is less than the typical pore size. For anisotropic materials, such as trabecular bones, the leading order behavior of $D(t)$ is linear in $\sqrt{D_{0} t}$ :

$$
\frac{D(t)}{D_{0}} \approx 1-\frac{4}{3 \sqrt{\pi}}\left(\frac{S}{V}\right)_{p} \sqrt{D_{0} t},
$$

where $(S / V)_{p}$ is the projected SVR $\left(P S V R_{P F G}\right)$ along a certain direction (40). This relationship holds regardless of the detailed microstructure of the restricting boundaries. Note that this formula applies for 1D diffusion along the chosen direction and does not assume an isotropic diffusion behavior. The $P S V R_{P F G}$ quantity is analogous to the ADC in an anisotropic medium along the applied gradient direction $\hat{g}$, given by $A D C=\hat{g}^{\dagger} \cdot \hat{D} \cdot \hat{g}$, where $\stackrel{\leftrightarrow}{D}$ is the diffusion tensor (DT). We measured the time dependence of the ADC and extract PSVR $R_{P F G}$ along different directions.

For our measurements, the largest diffusion length $\sqrt{D_{0} t}$ $\sim 80 \mu \mathrm{m}$, was significantly smaller than the average pore size of $1 \mathrm{~mm}$ shown in the $\mu \mathrm{CT}$ images. Thus, the full ADC dataset is well approximated by Eq. [3], and a linear relationship between ADC and $\sqrt{D_{0} t}$ was observed for all samples. Finally, a residual $P S V R_{P F G, y}$ value $\left(0.55 \mathrm{~mm}^{-1}\right)$ due to the sample tube and the trapped water around the plugs was measured with bulk water in the same sample tube arrangement. This background was subtracted from the $P S V R_{P F G, y}$ data for bone samples. No residual $P S V R_{P F G, z}$ for bulk water was detectable within the experimental error. The error bars in $P S V R_{P F G}$ were estimated from the systematic error in the $D(t)$ measurement of the bulk water sample.

Given $P S V R_{P F G}$ values for six or more independent gradient directions, the full $(S / V)$ tensor can be determined (i.e., eigenvalues and eigenvectors) and a scalar measure of its anisotropy can be calculated. This measure is called the fractional anisotropy (FA) and is standard within DT imaging (DTI). Using the eigenvalues $\lambda_{i}$, the FA is defined by (41):

$$
F A=\sqrt{\frac{3}{2} \frac{\sum_{i=1}^{3}\left(\lambda_{i}-\Pi[\lambda]\right)^{2}}{\sum_{i=1}^{3} \lambda_{i}^{2}}},
$$

with $0<\mathrm{FA}<1$. In this case the FA was estimated with two assumptions: 1) alignment of the laboratory frame and principal frame (i.e., the measured PSVRs are the eigen- 
values), and 2) cylindrical symmetry (i.e., $P S V R_{P F G, y}=$ $\left.P S V R_{P F G, X}\right)$.These assumptions are reasonable for this sample set and will be supported by the $\mu \mathrm{CT}$ analysis shown further below. Note that this tensor is not equivalent to the standard DT, but instead reflects deviations of the water diffusion from the bulk value.

\section{$\mu C T$ Imaging and Analysis}

$\mu \mathrm{CT}$ images of all cleaned cylindrical samples were acquired at a $34-\mu \mathrm{m}$ isotropic resolution using a commercial instrument ( $\mu$ CT 20, ScanCo Medical, Switzerland). Image processing was performed to produce structural indices to be correlated with both the experimental NMR methods and the mechanical strength of the sample set. The first index chosen was the MIL $(9,11)$ (also known as the trabecular spacing), which is often used in the literature for $\mu \mathrm{CT}$ or MR images. The first step was a binarization of the raw images into bone and water regions through a threshold technique. This was done using publicly available software (ImageJ) employing the iterative isodata algorithm, which uses the average of two averages taken over pixels above and below one iteration's threshold as the next iteration's value until convergence is reached. Then, test lines were considered through every voxel in the sample along a given direction, and the lengths of segments between regions of bone were averaged over a volume entirely within the bone structure to avoid edge effects. This process was carried out here for the three Cartesian axes of the image $(x, y, z)$.

The second index calculated from the images was a projected surface to volume ratio $\left(P S V R_{\mu C T}\right)$ along each of the coordinate axes $(x, y, z)$. Beginning with the binarized images, the surface area was calculated as the number of exposed voxel faces $N_{S}$ along the test direction (i.e., interfaces between bone and marrow space). Similarly, the volume was estimated by counting the number of voxels $N_{V}$ in the marrow space within the considered volume. The voxel dimension $\Delta x=34 \mu \mathrm{m}$ were used to calibrate the final ratio as $P S V R_{\mu C T}=\left(N_{S} / N_{V}\right) \Delta x^{-1}$.

The final index calculated was the trabecular number $T b . N$, or the number of trabeculae per unit length. For this calculation, the binarized images were skeletonized, i.e., the finite thickness trabeculae were progressively eroded to a final configuration of $1 \mathrm{D}$ curves in space. This was performed with the ImageJ image processing software. Then, the number of trabeculae per unit length along a given direction $(x, y$, or $z$ ) was determined by averaging the lengths of voids between them, i.e., by performing an MIL calculation on the skeletonized images. Since this algorithm operates on 2D images, the reported Tb.N values for a particular direction $(x, y$, or $z)$ were derived from image orientations containing that direction.

\section{Internal Magnetic Field Calculation}

The $\mu$ CT images were used to calculate the spatial variation of the internal magnetic field for a few bone samples. The approach taken, guided by fundamental electromagnetic theory (42), is described in detail in Ref. 43 and is similar to that used in other work (10). In this method, the boundary between the two materials (in this case, bone and water) is assigned a "magnetic charge" density, $\rho_{m} \propto(\hat{n} \cdot \hat{d})$, where $\hat{n}$ is the local surface normal, and $\hat{d}$ is the applied field direction. The total internal field is then a sum of contributions from all surfaces:

$$
\frac{\vec{B}_{d}^{\text {int }}(\vec{r})}{B_{d}^{\text {applied }}}=\frac{\mu_{0} \Delta \chi}{4 \pi} \int_{S} \frac{\vec{r}-\vec{r}^{\prime}}{\left|\vec{r}-\vec{r}^{\prime}\right|^{3}}(\hat{n} \cdot \hat{d}) d S^{\prime},
$$

where $\Delta \chi=\chi_{\text {bone }}-\chi_{\text {water }}$ is the magnetic susceptibility difference of bone and water, and $\Delta \chi=-3 * 10^{-6}$ (MKS units) (10) was used for the present calculations. The applied field is $2 \mathrm{~T}$.

\section{RESULTS}

Figure 3 shows the salient features of the range of trabecular bone structures in this study, from weak (yield stress $=2.4 \mathrm{MPa}$, sample A) to intermediate (6.0 MPa, sample B) to strong (34.5 $\mathrm{MPa}$, sample C). Qualitative changes in the density, shape, and anisotropy of the trabecular elements are evident. The weakest sample shows a loosely connected network of mostly rods, the intermediate sample shows a more entangled structure of rods and plates, and the strongest sample consists mainly of highly

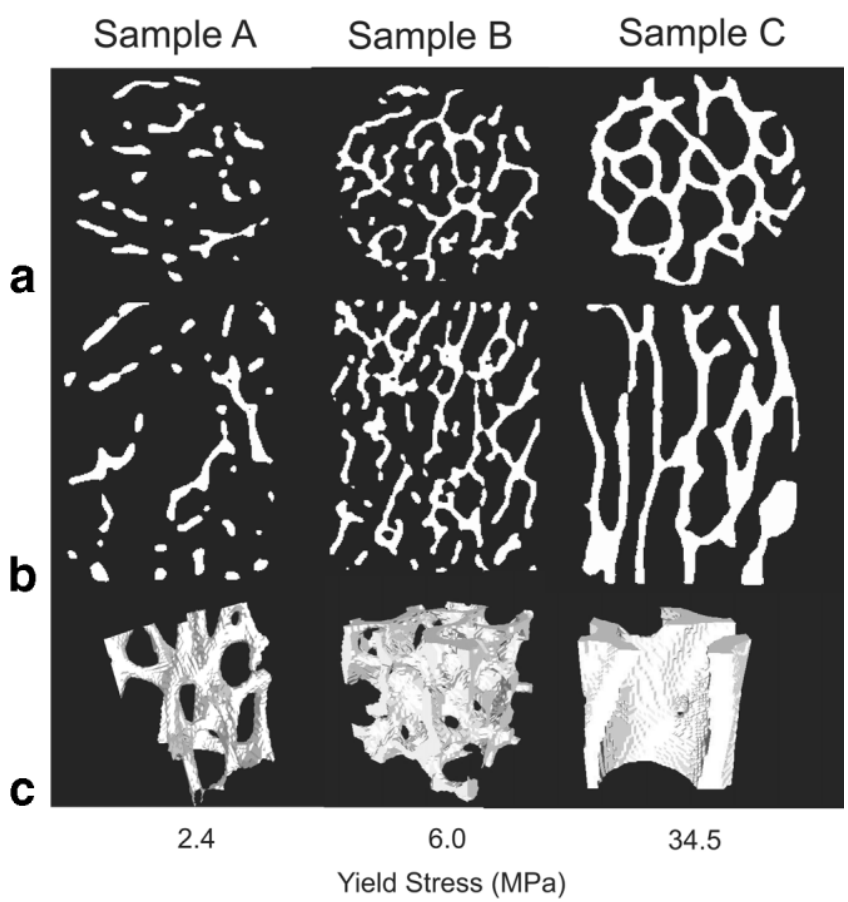

FIG. 3. $\mu C T$ images of three representative trabecular bone samples showing the full range of trabecular structures for this study: (a) transverse slice, (b) longitudinal slice, and (c) 3D rendering of a cubical subvolume at the center of each sample $(2 \times 2 \times 2 \mathrm{~mm})$. Images were acquired at an isotropic resolution of $34 \mu \mathrm{m}$. The samples shown cover the range of mechanical yield stresses in the present study, from weak (2.4 MPa, sample A) to intermediate (6.0 MPa, sample B) to strong (34.5 MPa, sample C). The structures show a progression from interconnected rods (sample A) to an entangled combination of rods and plates (sample B) to a set of highly oriented plates (sample C). 
oriented plates with few perforations. The results of the experimental techniques (NMR, $\mu \mathrm{CT}$ ) are discussed in more detail individually below.

\section{DDIF NMR}

Figure 4a and b show the results of the DDIF and reference experiment for the three samples shown in Fig. 3. In the DDIF magnetization decay data (Fig. 4a), the initial decay rate is larger for sample $\mathrm{B}$ compared to the other two, while the reference data (reflecting spin-lattice relaxation) are both single-exponential and identical for all three samples, showing a time constant of $T_{1}=2.5 \mathrm{~s}$. In the Laplace inversion spectra (Fig. 4b), there is a correspondingly higher spectral weight in the short-decay time region for sample B compared to the other two. This dependence for the full range of samples in this study is shown in Fig. 5. The weight in the fast decay region grows continuously from 2.4 to $6.0 \mathrm{MPa}$, and then falls as the bone strength increases further. This trend is shown graphically in Fig. $6 a$, where the integrated weight in the fast-decay region (20 ms $<T<500 \mathrm{~ms}$ ) is shown as a function of yield stress.

\section{PFG NMR}

Figure 4c shows the results of the time-dependent diffusion coefficient measurements along two different gradient directions for samples A-C. Each shows a different amount of restriction and/or anisotropy. The weakest sample (sample A) shows nearly unrestricted diffusion along both directions, consistent with its high porosity. Sample B shows restricted diffusion in both directions, with a more pronounced transverse than longitudinal restriction. Finally, sample $\mathrm{C}$ shows restriction mainly transverse to the sample axis, giving it the maximum anisotropy of the three.

As described in the Materials and Methods section, $P S$ $V R_{P F G}$ was extracted from each $D(t)$ dataset for both the longitudinal and transverse directions. Both datasets ( $P S$ $V R_{P F G, y}, P S V R_{P F G, z}$ ) are plotted along with the DDIF data as a function of mechanical yield stress in Fig. 6a. The strong correlation between DDIF and $P S V R_{P F G, z}$ is evident in both the low-and high-strength regimes. The lower panel shows the FA of the $P S V R_{P F G}$ tensor, calculated using Eq. [4]. Two regimes of strength are evident: a weak regime (yield stress (YS) $<7 \mathrm{MPa}$ ) with a roughly constant anisotropy, and a strong regime (YS > $7 \mathrm{MPa}$ ) with an increasing anisotropy with yield stress. These regimes are highlighted with guides to the eye in Fig. 6a.

\section{$\mu \mathrm{CT}$ Image Analysis}

The results of the MIL calculation for the entire sample set, displayed as inverse lengths for comparison with NMR data, are shown in Fig. 7a. The inverse MIL values for the two transverse directions $\left(\left(M I L_{x}\right)^{-1}\right.$ and $\left.\left(M I L_{y}\right)^{-1}\right)$ show a similar trend that increases in the weak bone regime and saturates to a constant in the strong regime, consistent with the trend in $P S V R_{P F G, y}$. The longitudinal $\left(M I L_{z}\right)^{-1}$ values are lower than the transverse values for all samples, and show an increasing trend for the weak regime but a decreasing trend for the strong regime. The trends are similar to those measured with the NMR diffusion techniques (Fig. 6a), and are similarly indicated with dashed lines in Fig. 7a.

Figure $7 \mathrm{~b}$ shows the calculated SVRs $P S V R_{\mu C T}$, scaled by a factor of 2 for quantitative comparison with the other $\mu \mathrm{CT}$ indices. Very similar trends to those in the MIL data
FIG. 4. Representative data from NMR experiments on samples A-C. a: NMR DDIF and reference signal decay as a function of diffusion time $t_{D}$. b: NMR DDIF spectra as a function of decay time constant $T$ due to diffusion in the internal field. c: Time-dependent diffusion coefficients along two different directions measured with the sequence in Fig. 2.

\section{Sample A Sample B Sample C}
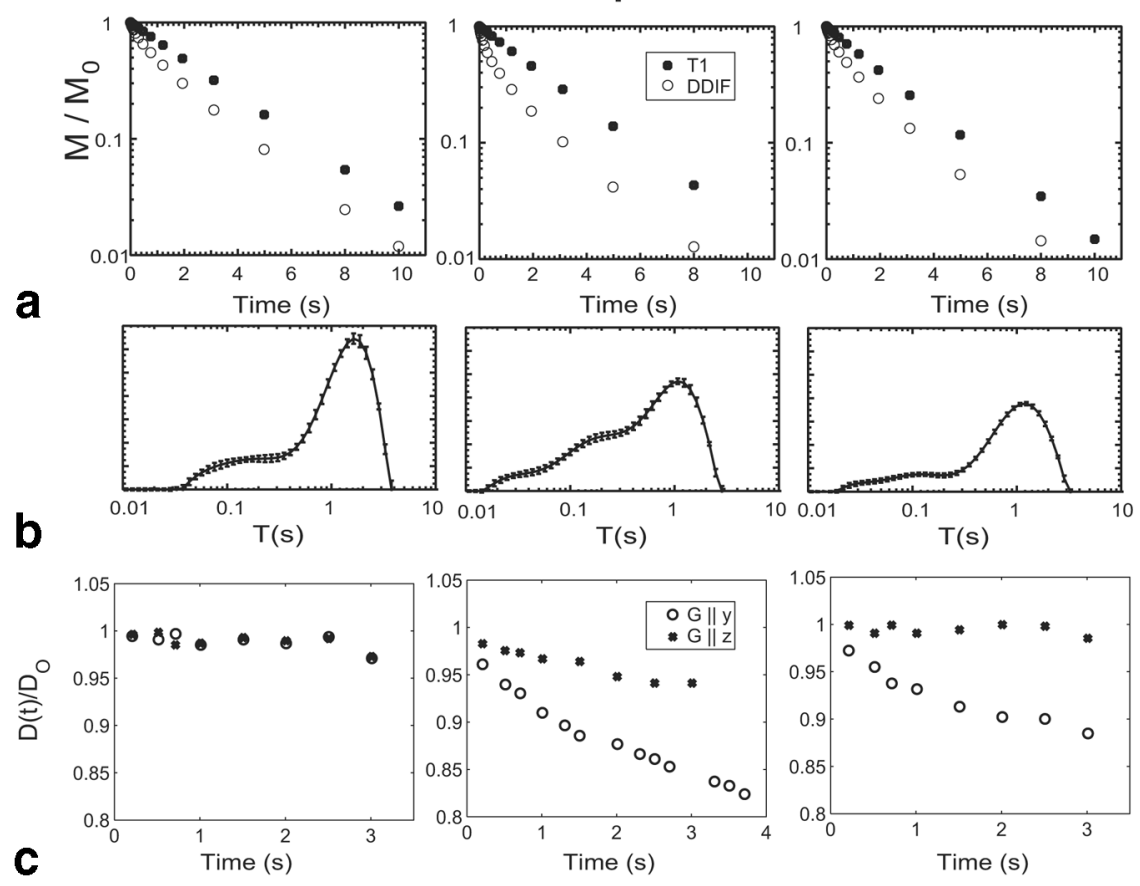


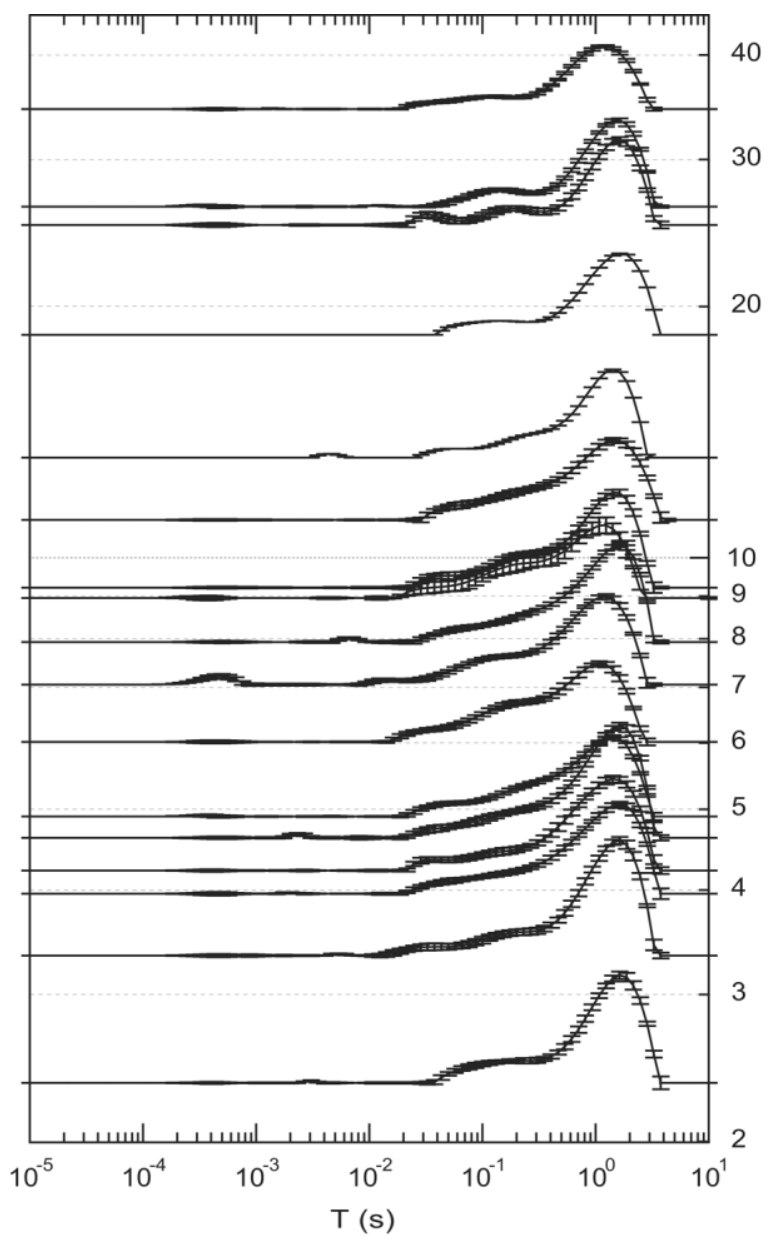

FIG. 5. DDIF spectra for all bovine bone samples in the present study. The spectra are shifted vertically proportional to the sample's yield stress.

are evident in the $P S V R_{\mu C T}$ datasets, in that all three indices increase with strength in the weak regime, the transverse indices saturate in the strong regime, and the longitudinal index decreases with strength in the strong regime. The quantitative values are also very close to their MIL counterparts. Figure 7c shows the trabecular number indices $T b . N$ for each of the three directions $(x, y, z)$. Again, they show similar behavior to the other two $\mu \mathrm{CT}$ index sets, but with a somewhat compressed dynamic range and lower values.

\section{Internal Magnetic Field Calculation}

Figure 8 shows results of the internal magnetic field calculation for the three representative samples (samples A-C). Isosurfaces of magnetic field at a field offset of $\Delta v=$ $25 \mathrm{~Hz}(\Delta \mathrm{B}=6 \mathrm{mG}$, or about $0.3 \mathrm{ppm}$ at $2 \mathrm{~T})$, are shown in red. The calculated distribution for sample $\mathrm{C}$ showed a significant $(16 \mathrm{~Hz})$ spectral offset to lower frequencies. Though it is structure-dependent, this shift does not change the local gradients and is thus not of primary interest in this work. For qualitative comparison, the displayed spectrum for sample C in Fig. 8 has been shifted to center on its peak frequency, and the displayed isosurface is that occurring $\Delta v=25 \mathrm{~Hz}$ above this peak frequency. In all three cases, the regions of highly deviated field are found to exist in close proximity to the trabecular surfaces, in particular near those oriented perpendicular to the applied field. Furthermore, the abundance of these surfaces and corresponding spectral intensity are both maximal for the intermediate strength sample (sample B).

\section{Correlations}

A vital characteristic of an experimental indicator of fracture risk is its correlation with mechanical strength. In the present work several such indicators have been presented from both NMR experiments and analysis of $\mu \mathrm{CT}$ image data. Table 1 shows the Pearson's correlation coefficients $r$ obtained from linear fits showing the correlation of the measurements with yield stress, BVF, and each other. Figure 9 shows one example fit between the DDIF data and $P S V R_{P F G, Z}$, which showed one of the highest correlations of the study $(r=0.84)$. The lowest rows show the correlation coefficient $r$ (two significant digits) and zero correlation probability $p$ (one significant digit) of a multilinear regression of yield stress vs. a linear combination of the BVF and another index. This approach, which has been applied in the literature for other microstructural measures (11), employs the new indices in a supplementary role to the BVF
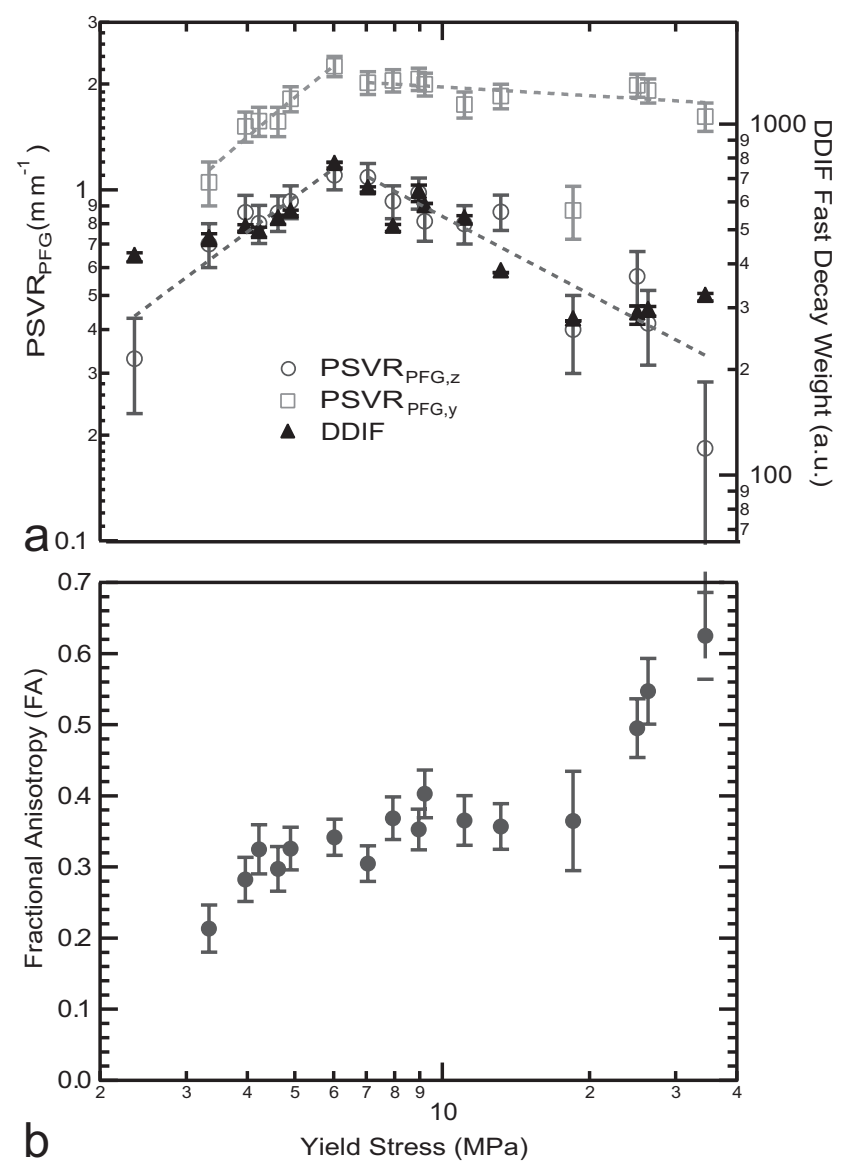

FIG. 6. a: Plot of NMR results (DDIF and PSVR) as function of yield stress, showing a highly correlated DDIF result with $\mathrm{PSVR}_{\mathrm{z}}$. b: Plot of the FA as a function of yield stress. 
FIG. 7. Plot of three structural indices calculated from $\mu \mathrm{CT}$ images for the trabecular bone sample set as a function of yield stress, and for three perpendicular orientations. The indices are (from top to bottom): MIL, PSVR, and Tb.N.

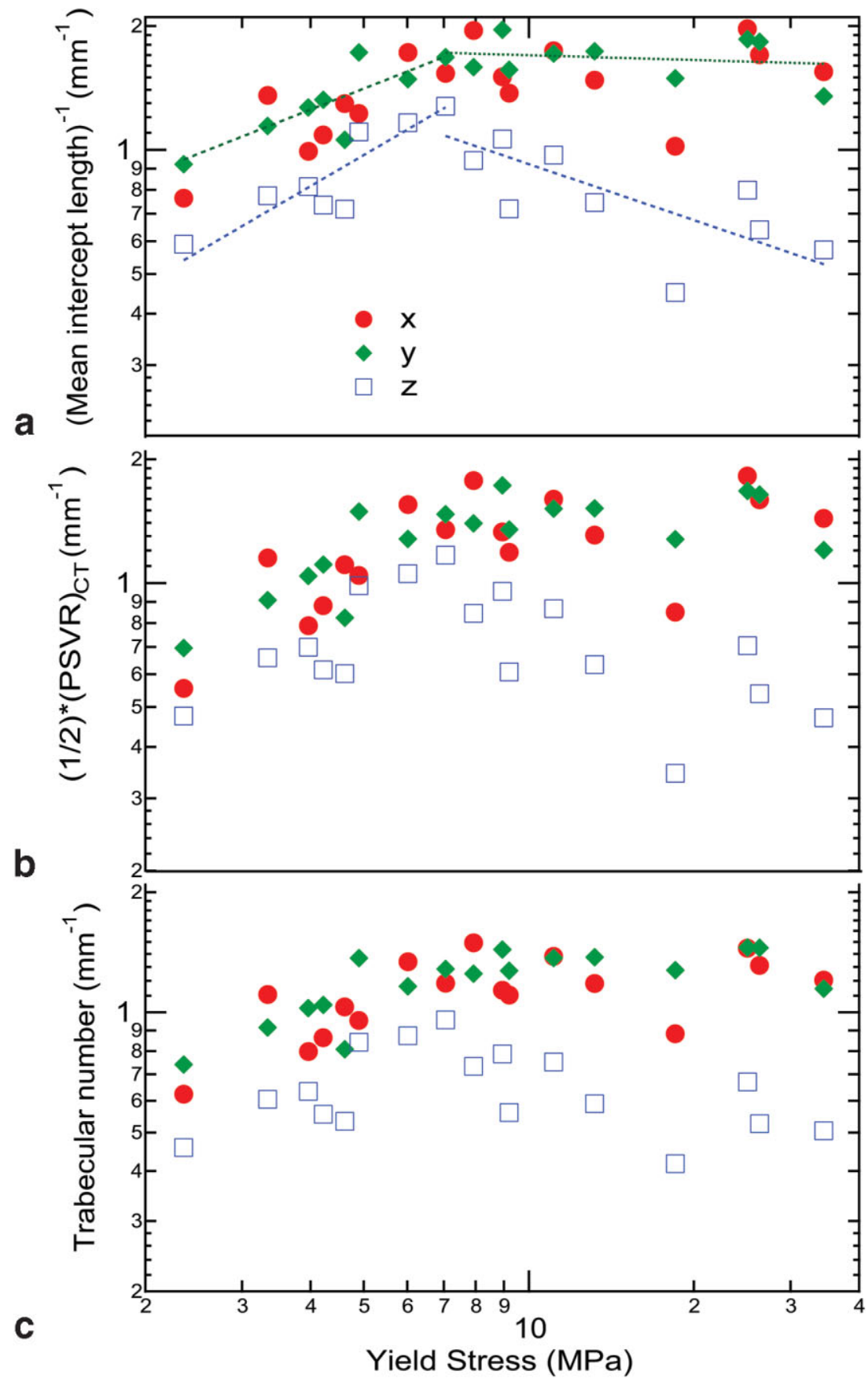

information. The largest increase over the single BVF-yield stress correlation is afforded by the inclusion of the PSVR${ }_{P F G, Z}$ data. Figure 10 depicts this improvement with plots of the correlations of yield stress with BVF (Fig. 10a) and the optimal linear combination of BVF and $P S V R_{P F G, Z}$ (Fig. 10b).

\section{DISCUSSION}

The various techniques applied to this sample set demonstrated quite consistent results, and when taken together provide a clearer understanding of the DDIF contrast. The agreement of the DDIF and $P S V R_{P F G, z}$ data in Figs. 6 and 9 and the similarity with the $\left(M I L_{z}\right)^{-1}, P S V R_{\mu C T, z}$, and Tb.N.z data in Fig. 7 are clear indications of DDIF's sensitivity to the PSVR of trabecular bone. The orientation of sensitivity for the DDIF experiment is set by the direction of the applied field. Consistently, the internal field calculation in Fig. 8, in agreement with intuition and previous work (10), shows that the magnetic field deviates from its average in regions close to the trabecular surfaces perpendicular to the applied field. Since these extremal field values are accompanied by large internal field gradients, it is reasonable to expect these regions to dominate the DDIF signal, making it an excellent method probe of PSVR along the field direction. For example, the abundance of highlighted regions is highest for the intermediate strength sample (sample B), consistent with its maximal value of PSVR. In comparison, the $T_{2}^{\prime}$ method $(5,44)$ measures the 


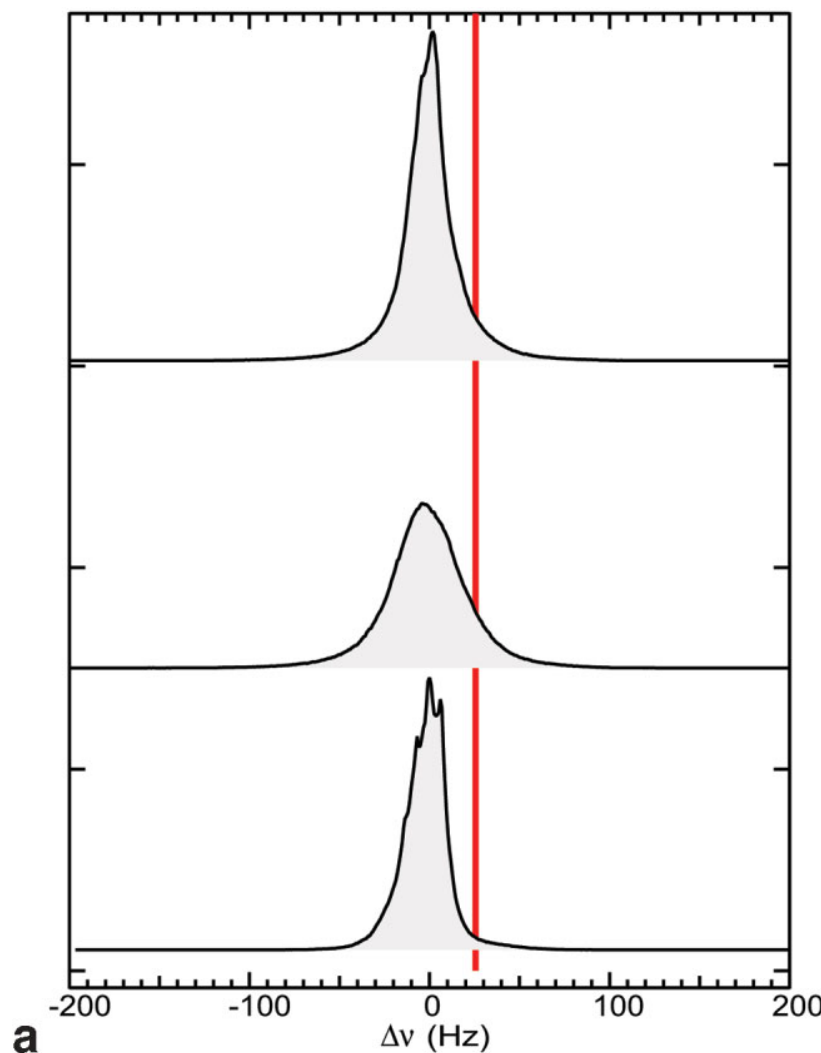

total field nonuniformity and may be more susceptible to macroscopic field inhomogeneities, such as imperfect shims (6).

Quantitative agreement exists among the experimental results as well. All longitudinal techniques (DDIF, PSVR${ }_{P F G, z},\left(M I L_{z}\right)^{-1} P S V R_{\mu C T, z}$, and Tb.N.z ) show a dynamic range over the whole sample set of approximately a factor of 2. Those that are calibrated also display approximately the same absolute values $\left(0.5<P S V R<1.0 \mathrm{~mm}^{-1}\right)$. The transverse measurements, $P S V R_{P F G, y}\left(M I L_{x, y}\right)^{-1}, P S V R_{\mu C T, x, y}$,
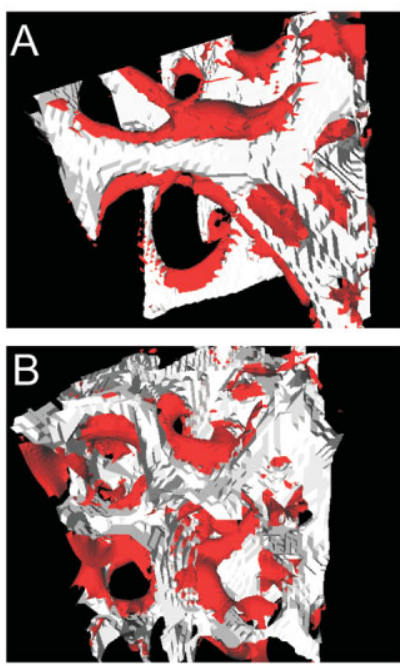

8. a: Internal magnetic field distribution for samples A-C calculated from the $\mu \mathrm{CT}$ images. $\mathbf{b}$ : Isosurfaces of internal magnetic field at $\Delta v=25 \mathrm{~Hz} \sim 6 \mathrm{mG} \sim$ $0.3 \mathrm{ppm}$ (marked as the vertical line in (a) are shown together with the trabecular structure subvolumes. Note that this deviated field value occurs near the trabeculae surfaces, in particular near those oriented perpendicular to the applied field (vertical in these images)

Table 1

Pearson's Correlation Coefficients $(r)$ Between Experimental Probes of Trabecular Bone*

\begin{tabular}{|c|c|c|c|c|c|c|c|c|c|c|c|c|c|}
\hline & BVF & DDIF & $P S V R_{z, P F G}$ & $\left(M I L_{z}\right)^{-1}$ & $P S V R_{z \mu C T}$ & Tb.N.Z & $P S V R_{y, P F G}$ & $\left(M I L_{y}\right)^{-1}$ & $P S V R_{y \mu C T}$ & Tb.N.y & $\left(M I L_{x}\right)^{-1}$ & $P S V R_{x \mu C T}$ & Tb.N.X \\
\hline YS & 0.91 & -0.68 & -0.65 & -0.41 & 0.48 & -0.31 & -0.00 & 0.38 & 0.44 & -0.48 & 0.43 & 0.48 & -0.42 \\
\hline BVF & & -0.52 & -0.37 & -0.12 & -0.09 & -0.01 & 0.23 & 0.60 & 0.65 & 0.67 & 0.68 & 0.72 & 0.67 \\
\hline DDIF & & & 0.84 & 0.80 & 0.79 & 0.73 & 0.48 & 0.02 & -0.01 & -0.09 & 0.06 & 0.02 & 0.06 \\
\hline$P S V R_{z, P F G}$ & & & & 0.83 & 0.82 & 0.78 & 0.49 & 0.27 & 0.23 & 0.16 & 0.22 & 0.17 & 0.21 \\
\hline$\left(M I L_{z}\right)^{-1}$ & & & & & 1.00 & 0.99 & 0.63 & 0.42 & 0.40 & 0.31 & 0.40 & 0.36 & 0.36 \\
\hline$P S V R_{z, \mu C T}$ & & & & & & 0.99 & 0.64 & 0.44 & 0.42 & 0.33 & 0.42 & 0.39 & 0.39 \\
\hline Tb.N.Z & & & & & & & 0.63 & 0.49 & 0.47 & 0.39 & 0.46 & 0.43 & 0.43 \\
\hline$P S V R_{y, P F G}$ & & & & & & & & 0.58 & 0.59 & 0.48 & 0.65 & 0.63 & 0.59 \\
\hline$\left(M I L_{y}\right)^{-1}$ & & & & & & & & & 0.99 & 0.98 & 0.64 & 0.65 & 0.61 \\
\hline$P S V R_{y, \mu} C T$ & & & & & & & & & & 0.99 & 0.67 & 0.68 & 0.64 \\
\hline Tb.N.y & & & & & & & & & & & 0.61 & 0.63 & 0.60 \\
\hline$\left(M I L_{x}\right)^{-1}$ & & & & & & & & & & & & 1.00 & 0.99 \\
\hline$P S V R_{x \mu C T}$ & & & & & & & & & & & & & 0.99 \\
\hline$A^{\star} \mathrm{BVF}+$ & & & & & & & & & & & & & \\
\hline$B^{\star}$ index & & 0.94 & 0.97 & 0.96 & 0.96 & 0.96 & 0.94 & 0.93 & 0.93 & 0.92 & 0.95 & 0.94 & 0.94 \\
\hline$P / 10^{-7}$ & 4 & 0.2 & 0.0009 & 0.01 & 0.009 & 0.01 & 0.6 & 0.5 & 0.6 & 1 & 0.08 & 0.2 & 0.2 \\
\hline
\end{tabular}

${ }^{*}$ Next-to-last row: Correlation coefficients with strength for multiple linear regressions with bone volume fraction (BVF) and one of the indices. Lowermost row : $P$-values (random correlation probability) for the multiple regression analyses. 


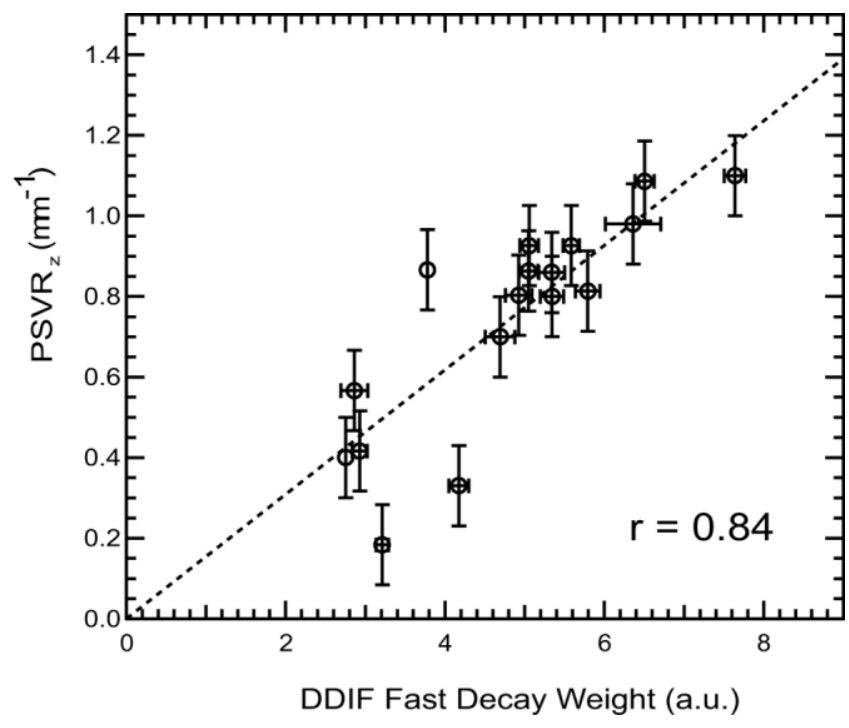

FIG. 9. Linear correlation between DDIF fast decay weight and $P S V R_{z}$.

row shows the single parameter correlation of all indices with yield stress. BVF provides the best correlation, underscoring the role of the other indices as supplementary information rather than replacement predictors. BVF correlates very poorly with the longitudinal indices (average $r=0.22$ ) and somewhat better with the transverse indices $(r=0.60)$. Apparently, the longitudinal indices provide nondensitometric information. When correlations among equidirectional indices are compared, good correlations are found $(r(z-z)=0.86, r(y-y)=0.77, r(x-x)=0.99)$. Comparing the two transverse $\mu \mathrm{CT}$ images also shows reasonable correlations $(r(x-y)=0.63)$, indicating the approximate cylindrical symmetry of these samples around their load-bearing axis $(z)$. Most importantly for this work, the DDIF contrast correlates well with all longitudinal indices, and most closely with $P S V R_{P F G, Z}(r=0.84)$. Given the anisotropy of the indices employed in this work, low correlations are expected when comparing indices along different directions. This is especially true when comparing longitudinal NMR quantities with transverse $\mu \mathrm{CT}$ indices $(r=0.13)$, and to a lesser extent when all longitudi- nal vs. transverse correlations are considered ( $r=0.33)$. In general, the correlations involving the three $\mu \mathrm{CT}$ indices are typically very similar, implying that they contain similar information. The trabecular number index (in which thickness information is absent) shows very slightly different results in some cases, e.g., a slightly worse correlation with DDIF. Evidently, the DDIF contrast probes the PSVR and is not a surface enumeration index. Finally, the multiple regression correlations with strength in the last row show the longitudinal information to be a bigger boon to strength prediction than the transverse data. This is consistent with mechanical arguments emphasizing the importance of reinforcing struts between trabecular plates (45). It should be noted that this sample set has a fairly high unsupplemented BVF-yield stress correlation $(r=$ 0.91). Future studies with a more polydisperse sample or subject batch may reinforce the conclusions drawn here.

In general, correlations between equidirectional NMR data $(r=0.84)$ or equidirectional $\mu \mathrm{CT}$ data $(r=0.99)$ are higher than those between equidirectional NMR and $\mu \mathrm{CT}$ data (longitudinal: $r=0.79$, transverse: $r=0.59$ ). One potential contribution to this discrepancy is a difference in effective resolution. The minimum probing length of the NMR measurements, given by the smallest diffusion length probed, is on the order of a few $\mu \mathrm{m}$, whereas the resolution of the $\mu \mathrm{CT}$ images is somewhat larger $(34 \mu \mathrm{m})$. This may give the experiments access to a more accurate and complete PSVR than that derived from the $\mu \mathrm{CT}$ images. However, an alternate possibility to this hypothesis is that the lower correlations from the $\mu \mathrm{CT}$ image analysis arise from the limitations in the present computational algorithms. Many sophisticated analysis techniques have been developed and demonstrated in the literature for bone microimaging (e.g., Refs. 9 and 12-14), and their application may improve the correlations obtained from this sample set.

The nonmonotonic dependence on strength shown in Fig. 6 for the two longitudinal NMR experiments (DDIF and $P S V R_{P F G, z}$ ) is noteworthy. It shows an initial increase with strength to an extremum that occurs at YS $\sim 7 \mathrm{MPa}$, and decreases for higher yield strengths. The same qualitative trend was obtained in the $\left(M I L_{\mathrm{z}}\right)^{-1}, P S V R_{\mu C T, z}$, and Tb.N.z data (Fig. 7). Qualitatively, these two regimes may be interpreted as follows: In the weak regime the amount
FIG. 10. Yield stress correlation plots. Left: Yield stress vs. BVF correlation. Right: Yield stress vs. (BVF, PSVR $R_{z, P F G)}$ correlation; coefficients $A$ and $B$ determined by multiple linear regression. The strength predictive ability of the combined parameter (right) exceeds that of the BVF alone.

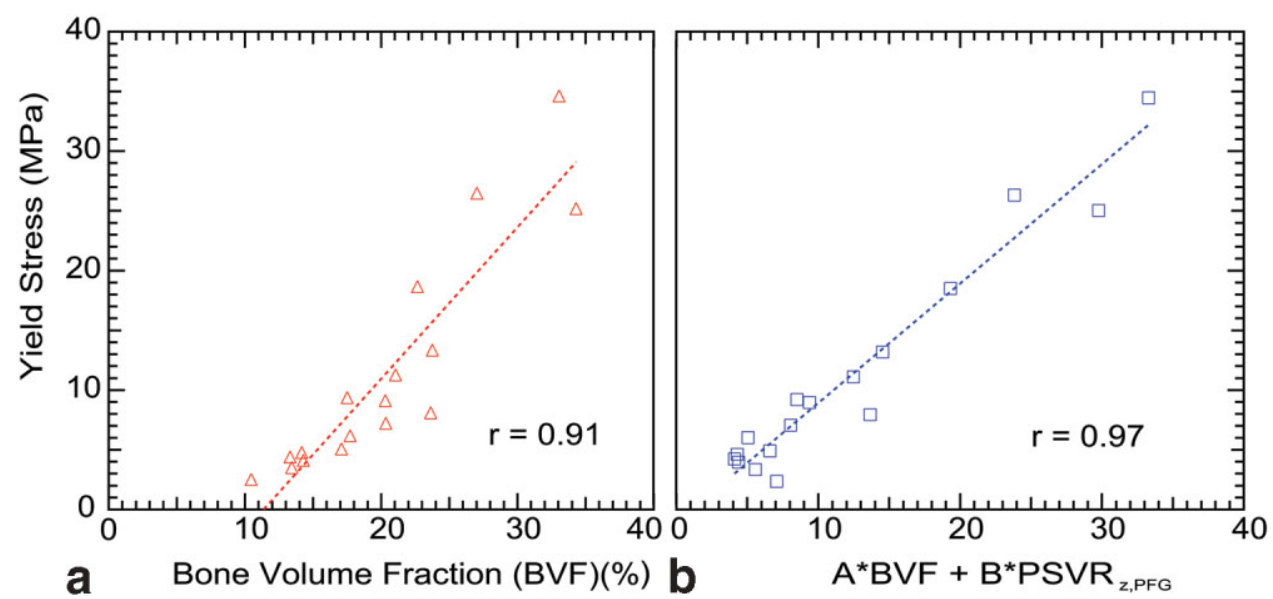


of bone material is relatively small, and the strength is controlled more by bone density than bone structure. In the stronger regime the samples can be more anisotropic, and their strength is more strongly affected by structural arrangement. The fact that the BVF-strength correlation was most improved with the inclusion of $P S V R_{P F G, \mathrm{Z}}$ data, which shows different behavior in the two regimes, supports this interpretation and is consistent with the loadbearing axis of these samples $(z)$. The origin of these two regimes within this sample set is more limited to speculation. Since the samples of variable strength are from different positions of the bovine tibia, the mechanical stress experienced by the samples during their growth can be substantially different. It is known that bone remodeling can be influenced by applied forces, and that the structural variation reflects different mechanical stress histories (46). The specimens of high strength may derive their highly anisotropic trabecular structure from the higher stress experienced during their growth, consistent with the numerical modeling of the stress-induced alignment of trabeculae (47). The stress-induced growth may not be significant for the low-strength samples due to inactivity or skeletal location, leaving them more susceptible to ongoing perforation. The resulting final structure then has much lower anisotropy.

While it is generally agreed that bone-quality issues are vital for improved fracture risk assessment, the variety of scalar indices employed to characterize this quality is quite broad and extends well beyond those considered here. The indices derived from microimaging techniques have a well-defined geometrical meaning, but those derived from bulk-averaged measurements, such as $T_{2}^{\prime}(5)$ and ultrasound (48), are more empirical. For the diffusionbased NMR measurements (DDIF and $D(t)$ ), the quantitative diffusion length $\left(\sqrt{D_{0} t_{D}}, \sqrt{D_{0} \Delta}\right)$ lends a solid geometrical basis to the measurement. In the $D(t)$ case this translates to a quantitative measure of the PSVR along the gradient direction. The DDIF signal is clearly controlled by this parameter, as demonstrated by the close agreement between the two measurements and the understanding of the origin of the internal field in trabecular bones. This is potentially useful given the close correlation between DDIF, $P S V R_{P F G, z},\left(M I L_{\mathrm{z}}\right)^{-1}$, and Tb.N.z, the latter of which is well known to be a powerful supplement to the densitystrength predictive relationship.

The quantitative interpretation of the full DDIF spectrum, though appealing from an informational standpoint, is complex. Structural properties, such as trabecular thickness, separation, and orientation, contribute to the spectrum differently. The large weighting of surfaces that are perpendicular to the field direction in the gradient distribution has already been noted. The relative contributions of each of these parameters to the decay rate distribution can only be fully elucidated through a detailed understanding of the internal field (e.g., Fig. 8) and the spin dynamics of the diffusing molecules $(6,10,27,28)$. Such calculations may indicate what further parameters can be extracted from DDIF data in a model-independent way. Furthermore, the quantitative dependence of the DDIF spectrum on the encoding time $t_{e}$ beyond the weak encoding limit should also be taken into account for analysis of the fastest decay components of the spectrum (29). These approaches may allow more detailed interpretation of the DDIF spectrum. However, if the goal is the PSVR information demonstrated by the partial moment of the DDIF spectrum, far less sampling are needed. Virtually the same trend vs. strength is obtained from decay rates derived from single exponential fits to the early time portion of the DDIF decay $\left(0<t_{D}<70 \mathrm{~ms}\right)$. This increases the feasibility of tests of the DDIF contrast in clinical scanners to evaluate its potential utility.

It is worth mentioning that to date, NMR diffusion measurements on trabecular bone have been rare $(49,50)$. Though such measurements are complicated by shorter relaxation times and smaller diffusivities due to the presence of marrow in vivo, the strongest reason for this rarity is the small accessible diffusion lengths $(10-100 \mu \mathrm{m}) \mathrm{com}-$ pared to the average pore size $(0.2-1 \mathrm{~mm})$. This makes the differences in diffusivities due to trabecular restrictions relatively small (Fig. 4c). Conventional diffusion-weighted imaging (DWI) or DTI would thus show limited contrast. However, if the slopes of the diffusion-time dependencies are compared from sample to sample, as in Fig. 4c, the relative contrast can be more striking. The cost of this enhanced contrast is the longer set of measurements required for the PSVR determination compared to a single ADC measurement. Another important consideration, to be addressed in future studies, involves the complicating aspects of bone marrow in the interpretation of DDIF data. Bone marrow's heterogeneous composition can lead to variations in susceptibility, diffusivity, and spin relaxation, which may interfere with the desired trabecular information. Both the diffusivity and the spin relaxation time $T_{1}$ are smaller in marrow than in bulk water, which reduces the DDIF technique's sensitivity. A high applied field may help counterbalance these effects, since DDIF decay rate scales quadratically with internal gradients and thus with the applied field. However, this can only be verified by experiment, so the practical clinical utility of the DDIF approach remains an open question that future studies will address.

\section{CONCLUSIONS}

New NMR experimental probes of trabecular bone structure have been demonstrated on bovine trabecular bone samples and compared with several established characterizations. Both methods employ measurement of magnetization decay due to diffusion in magnetic field gradients. In one case (DDIF) these gradients are in the variation of the internal field due to the susceptibility variation between bone and water, and in the other case $(D(t))$, applied field gradients are used to measure the time-dependence diffusion coefficient. Both experiments revealed a nonmonotonic dependence on the mechanical yield stress, a trend that is determined by the PSVR along the loadbearing axis of the trabecular bones. This trend was also found by three methods of $\mu \mathrm{CT}$ image analysis of the same samples.

\section{ACKNOWLEDGMENTS}

We thank Dr. Chi Hyun Kim for assistance in providing the trabecular bone samples, M. Moody for assistance in 3D 
visualization of the images and field calculations, and Dr. J. Babb for support with the statistical correlation analyses.

\section{REFERENCES}

1. Goldstein SA, Goulet R, McCubbrey D. Measurement and significance of three-dimensional architecture to the mechanical integrity of trabecular bone. Calcif Tissue Int 1993;53:S127-132, S132-123.

2. Kleerekoper M, Villanueva AR, Stanciu J, Rao DS, Parfitt AM. The role of three-dimensional trabecular microstructure in the pathogenesis of vertebral compression fractures. Calcif Tissue Int 1985;37:594-597.

3. Song Y-Q, Ryu S, Sen PN. Determining multiple length scales in rocks. Nature (Lond) 2000;406:178-181.

4. Wehrli FW, Song HK, Saha PK, Wright AC. Quantitative MRI for the assessment of bone structure and function. NMR Biomed 2006;19:731764 .

5. Chung H, Wehrli FW, Williams JL, Kugelmass SD. Relationship between NMR transverse relaxation, trabecular bone architecture, and strength. Proc Natl Acad Sci USA 1993;90:10250-10254.

6. Engelke K, Majumdar S, Genant HK. Phantom studies simulating the impact of trabecular structure on marrow relaxation time, T2'. Magn Reson Med 1994;31:380-387.

7. Newitt DC, Majumdar S, Jergas MD, Genant HK. Decay characteristics of bone marrow in the presence of a trabecular bone network: in vitro and in vivo studies showing a departure from exponential behavior. Magn Reson Med 1996;35:921-927.

8. Yablonskiy DA, Reinus WR, Stark H, Haacke EM. Quantitation of T-2' anisotropic effects on magnetic resonance bone mineral density measurement. Magn Reson Med 1997;37:214-221.

9. Chung HW, Wehrli FW, Williams JL, Kugelmass SD, Wehrli SL. Quantitative-analysis of trabecular microstructure by 400-Mhz nuclear-magnetic-resonance imaging. J Bone Miner Res 1995;10:803-811.

10. Hwang SN, Wehrli FW. The calculation of the susceptibility-induced magnetic field from 3D NMR images with applications to trabecular bone. J Magn Reson B 1995;109:126-145.

11. Majumdar S, Kothari M, Augat P, Newitt DC, Link TM, Lin JC, Lang T, Lu Y, Genant HK. High-resolution magnetic resonance imaging: threedimensional trabecular bone architecture and biomechanical properties. Bone 1998;22:445-454.

12. Pothuaud L, Majumdar S. Simulation of the evolution of trabecular bone structure from high resolution magnetic resonance imaging: a preliminary study using "distance to topological fracture." J Bone Miner Res 2002;17:S414-S415.

13. Wehrli FW, Hwang SN, Ma JF, Song HK, Ford JC, Haddad JG. Cancellous bone volume and structure in the forearm: Noninvasive assessment with MR microimaging and image processing. Radiology 1998; 206:347-357

14. Link TM, Majumdar S, Augat P, Lin JC, Newitt D, Lu Y, Lane NE, Genant HK. In vivo high resolution MRI of the calcaneus: differences in trabecular structure in osteoporosis patients. J Bone Miner Res 1998; 13:1175-1182.

15. Majumdar S, Genant HK, Grampp S, Newitt DC, Truong VH, Lin JC, Mathur A. Correlation of trabecular bone structure with age, bone mineral density, and osteoporotic status: in vivo studies in the distal radius using high resolution magnetic resonance imaging. J Bone Miner Res 1997;12:111-118.

16. Hwang SN, Wehrli FW. Subvoxel processing: a method for reducing partial volume blurring with application to in vivo MR images of trabecular bone. Magn Reson Med 2002;47:948-957.

17. Robson MD, Gatehouse PD, Bydder GM, Neubauer S. Human imaging of phosphorus in cortical and trabecular bone in vivo. Magn Reson Med 2004;51:888-892.

18. Capuani S, Curzi F, Alessandri FM, Maraviglia B, Bifone A. Characterization of trabecular bone by dipolar demagnetizing field MRI. Magn Reson Med 2001;46:683-689.

19. Bouchard LS, Wehrli FW, Chin C-L, Warren WS. Structural anisotropy and internal magnetic fields in trabecular bone: coupling solution and solid dipolar interactions. J Magn Reson 2005;176:27-36.

20. Wolff J. Das Gesetz der Transformation der Knochen. Berlin: A. Hirschwald; 1892.

21. Weinstein RS, Hutson MS. Decreased trabecular width and increased trabecular spacing contribute to bone loss with aging. Bone 1987;8: 137-142.

22. Parfitt AM. Implications of architecture for the pathogenesis and prevention of vertebral fracture. Bone 1992;13:S41-S47.
23. Parfitt AM, Mathews CHE, Villanueva AR, Kleerekoper M, Frame B, Rao DS. Relationships between surface, volume, and thickness of iliac trabecular bone in aging and in osteoporosis. J Clin Invest 1983;72:1396-1409.

24. Wehrli F, Vasilic B, Saha P, Wald M. Performance comparison of the spatial autocorrelation function and the mean intercept-length in the determination of trabecular bone anisotropy in the in vivo environment. In: Proceedings of the 14th Annual Meeting of ISMRM, Seattle, WA, USA, 2006 (Abstract 1284).

25. Gomberg BR, Saha PK, Wehrli FW. Topology-based orientation analysis of trabecular bone networks. Med Physics 2003;30:158-168.

26. Cowin S. The relationship between the elasticity tensor and the fabric tensor. Mechanics Mater 1985;4:137-147.

27. Audoly B, Sen PN, Ryu S, Song Y-Q. Correlation functions for inhomogeneous magnetic field in random media with application to a dense random pack of spheres. J Magn Reson 2003;164:154-159.

28. Song Y-Q. Using internal magnetic fields to obtain pore size distributions of porous media. Concepts Magn Reson 2003;18A:97-110.

29. Lisitza NV, Song YQ. The behavior of diffusion eigenmodes in the presence of internal magnetic field in porous media. J Chem Physics 2001;114:9120-9124.

30. Hurlimann MD, Helmer KG, Sotak CH. Dephasing of Hahn echo in rocks by diffusion in susceptibility-induced field inhomogeneities. Magn Reson Imaging 1998;16:535-539.

31. Zhong J, Kennan RP, Gore JC. Effects of susceptibility variations on NMR measurements of diffusion. J Magn Reson 1991;95:267.

32. Majumdar S, Gore JC. Studies of diffusion in random fields produced by variations in susceptibility. J Magn Reson 1988;78:41-55.

33. Borgia GC, Brown RJS, Fantazzini P. The effect of diffusion and susceptibility differences on T2 measurements for fluid in porous media and biological tissues. Magn Reson Imaging 1996;14:731-736.

34. Norris DG. The effects of microscopic tissue parameters on the diffusion weighted magnetic resonance imaging experiment. NMR Biomed 2001;14:77-93.

35. Clark CA, Barker GJ, Tofts PS. An in vivo evaluation of the effects of local magnetic susceptibility-induced gradients on water diffusion measurements in human brain. J Magn Reson 1999;141:52-61.

36. Kim CH, Mikhail G, von Stechow D, Muller R, Guo XE. Effects of thresholding techniques in micro-CT based finite element models of trabecular bone. J Biomed Eng 2007; in press.

37. Tikhonov AN, Arsenin VY. Solutions of ill-posed problems. Halstead Press, New York; 1977

38. Williams WD, Seymour EFW, Cotts RM. A pulsed-gradient multiplespin-echo NMR technique for measuring diffusion in the presence of background magnetic field gradients. J Magn Reson 1978;31:271-282.

39. Callaghan PT. Principles of nuclear magnetic resonance microscopy. Oxford: Oxford University Press; 1993. 492 p.

40. Mitra PP, Sen PN, Schwartz LM. Short-time behavior of the diffusion coefficient as a geometrical probe of porous media. Phys Rev B 1993; 47:8565.

41. Basser PJ. Inferring microstructural features and the physiological state of tissues from diffusion-weighted images. NMR Biomed 1995;8:333-344.

42. Jackson JD. Classical electrodynamics. 2nd ed. John Wiley \& Sons, New York; 1962.

43. Sen PN, Audoly B, Axelrod S. Inhomogeneity in local magnetic field due to susceptibility contrast. J Appl Phys 1999;86:4548-4554.

44. Ma J, Wehrli FW. Method for image-based measurement of the reversible and irreversible contribution to the transverse relaxation rate. J Magn Reson 1996;111:61-69.

45. Mosekilde L. Vertebral structure and strength in-vivo and in-vitro. Calcif Tissue Int 1993;53:S121-S126.

46. Lanyon LE. Using functional loading to influence bone mass and architecture: objectives, mechanisms, and relationship with estrogen of the mechanically adaptive process in bone. Bone 1996;81:37-43S.

47. Huiskes R, Ruimerman R, van Lenthe GH, Janssen JD. Effects of mechanical forces on maintenance and adaptation of form in trabecular bone. Nature 2000;405:704-706.

48. Han S, Rho J, Medige J, Ziv I. Ultrasound velocity and broadband attenuation over a wide range of bone mineral density. Osteoporosis Int 1996;6:291-296.

49. Capuani S, Rossi C, Alesiani M, Maraviglia B. Diffusion tensor imaging to study anisotropy in a particular porous system: the trabecular bone network. Solid State Nucl Magn Reson 2005;28:266.

50. Ward R, Caruthers S, Yablon C, Blake M, DiMasi M, Eustace S. Analysis of diffusion changes in posttraumatic bone marrow using navigatorcorrected diffusion gradients. AJR Am J Roentgenol 2000;174:731-734. 